\title{
Noch weit vom Mainstream entfernt
}

\author{
Geschlechtliche Gleichstellung ist eine Querschnittsaufgabe für alle Politik- \\ felder, auch für Verbraucherpolitik. Aber warum sollten Geschlechterperspek- \\ tiven im Verbraucherschutz berücksichtigt werden? In welcher Hinsicht sind sie \\ relevant für eine geteilte Verantwortung?
}

$\mathrm{D}$

Von Irmgard Schultz er rechtliche und politikstrategische Rahmen für die Berïcksichtigung von Geschlechterperspektiven im Verbraucherschutz ist die politische Strategie des Gender Mainstreaming, die abkürzend als Integration der Geschlechterperspektive in alle Politikbereiche gekennzeichnet werden kann. 1999 hat die Europäische Union das Gender Mainstreaming im Vertrag von Amsterdam als eine rechtsverbindliche Querschnittsaufgabe festgeschrieben. Die Durchsetzung der „tatsächlichen Gleichberechtigung von Frauen und Männern“ ist auch Teil des Koalitionsvertrags der Bundesregierung. Im Juli 2000 wurde die Gemeinsame Geschäftsordnung aller bundesdeutschen Ministerien (GGO) dahingehend geändert, dass das Gender Mainstraming zum durchgängigen Leitprinzip der Bundesministerien bestimmt wurde. Für eine Berücksichtigung des Gleichstellungsauftrages im Verbraucherschutz bedarf es allerdings einiger Vorüberlegungen, um deren Notwendigkeit aufzuzeigen.

Die holländischen „Erfinderinnen“ des Instruments Gender Impact Assessment (GIA), M. Verloo und C. Roggeband, haben zwei gesellschaftliche Bereiche identifiziert, die ihrer Analyse nach immer genderrelevant sind. Das ist zum einen die geschlechtsspezifische Arbeitsteilung und zum anderen die gesellschaftliche Organisation von Intimität, $\mathrm{zu}$ dem zentral die Gesundheit, die menschliche Reproduktion, der Schutz der freien Beziehungswahl und der Privatsphäre zählt. Als einen dritten genderrelevanten Bereich hat das Institut für sozial-ökologische Forschung darüber hinaus die Gestaltungsmacht von Frauen und Männern in Politik, Wissenschaft und Technik hinzugefügt. Sie wurde exemplarisch entwickelt für die Durchführung eines GIA des Umweltforschungsprogramms der EU (2). Wie die drei Genderdimensionen, konkreter vorstellbar sind und welche Geschlechterperspektiven sie für den Verbraucherschutz begründen, soll im Folgenden kurz verdeutlicht werden.

\section{Geschlechtsspezifische Arbeitsteilung}

Hat eine politische Maßnahme des Verbraucherschutzes Auswirkungen auf die geschlechtsspezifische Arbeitsteilung? Die Frage, die diese Genderperspektive im Verbraucherschutz begründet, richtet sich einerseits auf die ungleiche Arbeits-, Zeit- und Ressourcenverteilung zwischen den Geschlechtern, andererseits aber auch auf einen ungleichen Zugang zu (qualifizierten) Arbeitsbereichen, zu Zeitprivilegien und zu Ressourcen. Bei dieser Frage kommt zuerst einmal der ganze Komplex ungleich verteilter Haus-, Versorgungs- und gesundheitlicher Vorsorgearbeit in den Blick, der die höchst unzulänglich geteilte Verantwortung für die unbezahlten Hausarbeiten zwischen Frauen und Männern betrifft.

Fragen wir nun, ob eine verbraucherpolitische Maßnahme, wie beispielsweise die Einführung der Kennzeichnungspflicht für gentechnisch veränderte Lebensmittel, Frauen und Männer

\section{Inserieren Sie in Ökologisches Wirtschaften!}

Sprechen Sie genau Ihre Zielgruppe an. Gesamtauflage von 1.500 Heften. Erscheint $5 \times$ jührlich.

Fordern Sie noch heute die Mediadaten an! Frau Katja Muchow berät Sie gerne.

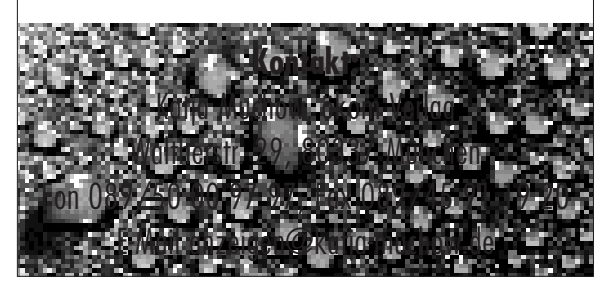

unterschiedlich betrifft und ob diese Maßnahme die geschlechtsspezifische Arbeitsteilung im Haushalt abbaut oder verstärkt. Zur Beantwortung dieser Frage muss geklärt werden: Wer kauft mehrheitlich ein? Wer hat angesichts der Doppelbelastungen in Berufs- und Familienarbeit weniger Zeit, sich über Qualitätsaspekte der Lebensmittel zu informieren? Wer hat hingegen häufiger Zugang zum Internet, um sich schnell und unaufwendig über die Gentechnikdebatte zu erkundigen?

Ein ernstes Problem ist die unzureichende Datenlage. Noch fehlen beispielsweise geschlechtsspezifisch aufgeschlüsselte Daten über die Internetnutzung von Verbraucherinformationen.

Darüber hinaus zeigt das Beispiel ein grundsätzliches Dilemma des Verbraucherschutzes, das die geteilte Verantwortung zwischen staatlichem Verbraucherschutz und häuslicher Versorgungsarbeit betrifft: Angesichts des Nichtwissens über viele Wirkungen von Produkten wie hier beispielsweise von Produkten mit gentechnisch veränderten Organismen und angesichts der Zulassung von ,unbedenklichen Restmengen“ unterhalb von Grenzwerten, die im Beispiel der gentechnisch veränderten Lebensmittel auf 0,9 Prozent festgelegt wurde, bleibt immer ein Restrisiko, mit dem umzugehen in Familienhaushalten mehrheitlich ein Problem der Frauen ist. Wie soll die Nichtexpertin die Kritik der Gentechnikkritiker an der Richtwert-Festsetzung der EU einschätzen? Wie kann sie beurteilen, ob die tolerierte Restgröße schädlich ist oder nicht? Sollte sie sich nicht doch noch weiter gehend informieren? Wo gibt es Informationen? Das Beispiel ist exemplarisch und kann auch auf andere Grenzwertfestsetzungen für Schadstoffe im Konsumbereich ausgedehnt werden: Frauen trifft die Unsicherheit über Verbraucherschutz-Informationen als Mehrarbeit bei der Informationsbeschaffung. Darüber hinaus bleibt das Restrisiko ihr Privatproblem. Zugespitzt könnte das so ausgedrïckt werden: Das Restrisiko wird privatisiert, die Informations- und Wissensermittlung hingegen feminisiert.

Nun kann der staatliche Verbraucherschutz an der Verwissenschaftlichung des Wissens über Gesundheitsrisiken von Produkten wenig ändern, hinsichtlich der Herstellung von Transparenz und alltagsnaher Information kann er jedoch neue Formen der ,,geteilten Verantwortung" in der Informationspolitik eingehen: beispielsweise indem das BMVEL die politische Auseinandersetzung in der EU über Grenzwert- 
und Restrisiko-Festsetzungen transparent macht und als Politikum kommuniziert. Die alltagsnahe Verbraucheraufklärung in den staatlich unterstützten Verbraucherzentralen hingegen sollte darüber hinaus Informationen zum alltagsnahen Umgang mit gentechnisch veränderten Lebensmitteln geben. Dabei müsste das Restrisiko kritisch zur Sprache kommen und die subjektiven Einschätzungen von privaten Frauen und Männern zu Restrisiken und einem adäquaten Umgang damit eruiert werden.

\section{- Gesellschaftliche Organisation von Intimität}

Hat eine politische Maßnahme des Verbraucherschutzes Auswirkungen auf die gesellschaftliche Organisation von Intimität? Die Frage, welche die zweite Genderperspektive im Verbraucherschutz begründet, richtet sich auf die unterschiedlichen Bedürfnisse und Ansprüche, die aus der physischen und psychosozialen Unterschiedlichkeit der Geschlechter und dem Schutz der Intimsphäre resultieren. Diese setzen jeder Auslagerungstendenz der Haus- und Versorgungsarbeit aus dem Haushalt heraus - und damit auch einem differenzlosen Gleichheitsverständnis - letztlich Grenzen.

Als ein Beispiel dafür kann die Auslagerung der Ernährungsverantwortung an öffentliche Versorger diskutiert werden. Konkret ist damit die durch das Verbraucherministerium angestoßene Diskussion und die Maßnahmen für eine bessere Gemeinschaftsverpflegung in Kindergärten oder Schulen etc. gemeint. Dies muss erst einmal als eine Entlastung der Privathaushalte und damit als ein Beitrag zur Entfeminisierung der Ernährungsverantwortung gewertet werden und ist daher aus Sicht der Geschlechtergerechtigkeit unbedingt zu begrüßen. Dennoch sind die Möglichkeiten der Auslagerung letztlich begrenzt. Das zu stillende Baby kann nicht beliebig durch externalisierte Nahrungsangebote ernährt werden und die psychosozialen Bedürfnisse von Müttern sollten berücksichtigt werden. Darüber hinaus ist bekannt, dass das sich ernähren auch aus gesellschaftliche Praktiken besteht, bei denen Beziehungen und Beziehungsfähigkeit entstehen. Dies begründet die große gesellschaftliche Bedeutung der gemeinsamen Mahlzeiten von Eltern und Kindern. Die Bedürfnisse der Mütter von zu stillenden Babies sind dank der Frauengesundheitsbewegung recht gut untersucht. Sie müssten als ein wichtiger Aspekt der gesellschaftlichen Organisation von
Intimität in den ernährungsbezogenen Verbraucherschutz aufgenommen werden, beispielsweise durch gezielte Kommunikationsstrategien des BMVEL an die Väter über eine sinnvolle Babyernährung und Möglichkeiten der Mütterentlastung.

Das Beispiel lenkt die Aufmerksamkeit auf einen wichtigen Punkt des Gender Mainstreaming. Gender ist kein neues Wort für Frauen. Was Bedürfnisse der Väter von Babies sind, ist angesichts des Fehlens einer Männergesundheitsbewegung kaum bekannt und nur durch neue Forschungen zu ermitteln. Als ein zentrales Problem der Männergesundheit, zu dem inzwischen auch in manchen Frauengesundheitszentren Beratungen angeboten werden, ist die zunehmende männliche Unfruchtbarkeit anzuführen. Die Frage nach den Wirkungen einer Verbraucherschutzmaßnahme auf die menschliche Gesundheit ist immer für die Männer- und Frauengesundheit bestimmter Alters- und Zielgruppen differenziert zu beantworten.

\section{Zur Gestaltungsmacht von Fraven und Männern}

Hat eine politische Maßnahme des Verbraucherschutzes Auswirkungen auf die Gestaltungsmacht von Frauen/ von Männern? Fördert oder minimiert sie ungleiche Machtbefugnisse? Diese dritte Genderperspektive im Verbraucherschutz richtet sich auf Frauen und Männer als Handelnde, als EntscheiderInnen und als Mitbestimmende. Für den öffentlichen Dienst sind wichtige Aspekte dieser Perspektive durch das Gleichstellungsgesetz im öffentlichen Dienst geregelt, in der Privatwirtschaft bisher nicht. Für das BMVEL ist festzustellen, dass die Besetzung des höchsten repräsentativen Amtes mit einer Frau von durchschlagender Bedeutung ist. Zu Recht kann Frau Künast behaupten: „Bislang waren Landwirtschaftsminister meist umfangreichere Herren mit dem entsprechenden Gebaren. Damit bedeutete meine Ernennung bereits - man könnte vielleicht sagen gestaltwahrnehmerisch - einen Kulturwandel, der jetzt für alle im wahrsten Sinne des Wortes ,sichtbar“ wird“ (1). Eine Frau auf oberster Entscheidungsebene eines Ministeriums ist neu und durchbricht die ,gläserne Decke“ der obersten Chefetage, in die Frauen sonst selten hinein kommen.

In der aktuellen Landwirtschafts- und Verbraucherpolitik stellt sich über den Aspekt der Geschlechtergleichstellung in den Entscheidungshierarchien hinaus die Frage nach der
Gestaltungsmacht von Frauen und Männern in möglichen Interessens-Allianzen neu. Denn insbesondere für eine Agrarwende sind neue Partnerschaften zu bilden, die das Verbraucherministerium im ,,magischen Sechseck von Landwirten, Futtermittelherstellern, Lebensmittelindustrie, Einzelhandel, Verbrauchern und Politik" sieht. Dabei sind die geschlechterspezifischen Aspekte und die Vertreterinnen für jede dieser sechs Interessensgruppen detailliert zu bestimmen. So behandelt das BMVEL, und das ist historisch neu, den Landfrauenverband als eine Interessensvertretungsgruppe mit eigenem Profil und nicht als Subgruppe des Bauernverbandes. Für die anderen fünf Interessensgruppen steht eine solche geschlechtsspezifische Differenzierung jedoch noch aus. Gäbe es ein solches ZwölfeckModell, würde dies vielleicht grundsätzlich oder in einzelnen Interessenpunkten zu anderen Partnerschaften führen als im Sechseck-Modell. Dieser politikstrategische Punkt des Gender Mainstreaming ist bisher jedoch nicht nur im Verbraucherschutz unterbelichtet. Grundsätzlich fehlen noch die best practice Beispiele von „good governance“, die zeigen, wie eine Politik des ,participatory decision-making“" unter systematischer Berücksichtigung von Frauen aussieht.

\section{Anmerkungen}

(1) Künast, R.: "Chancen gestalten" - über Macht - Strukturen - Möglichkeiten von Frauen. Rede auf dem 25. Herbsttreffen der Frauen in den Medien von ARD und ZDF, Potsdam, 2002.

(2) Schultz, I. et al.: Gender in Research - Gender Impact Assessment of the specific programms of the Fifth Framework Programm: Enviroment and Sustainable Development Sub-Programme. Brussels: European Commission, 2001.

\section{Die Autorin}

Irmgard Schultz ist Leiterin des Forschungsbereiches Alltagsökologie, Konsum und Stoffströme am Institut für sozial-ökologische Forschung (ISOE) Kontakt: ISOE, Hamburger Allee 45, 60486 Frankfurt. Tel. 069-7076919-16, E-Mail: Schultz@isoe.de 
(c) 20I0 Authors; licensee IÖW and oekom verlag. This is an article distributed under the terms of the Creative Commons Attribution Non-Commercial No Derivates License (http://creativecommons.org/licenses/by-nc-nd/3.o/), which permits unrestricted use, distribution, and reproduction in any medium, provided the original work is properly cited. 